\title{
Conformal Antenna Array for MIMO Applications
}

\author{
Taha A. Elwi1,2,3*, Z. Abbas' ${ }^{3}$, Mohammed Noori' ${ }^{1}$ Yahiea Al-Naiemy4, Ethar Y. Salih ${ }^{3}$, \\ Marwa M. Hamed ${ }^{2}$ \\ ${ }^{1}$ Department of Communication, Al-Mammon University Collage, Baghdad, Iraq \\ ${ }^{2}$ Department of Electronics and Communication, University of Baghdad, Baghdad, Iraq \\ ${ }^{3}$ Departmrnt of Physics, University Putra Malaysia, Serdang, Malaysia \\ ${ }^{4}$ Department of Computer Science, College of Science, University of Diyala, Diyala, Iraq \\ Email: ${ }^{*}$ taelwi@ualr.edu
}

Received 27 January 2014; revised 21 February 2014; accepted 11 March 2014

Copyright (C) 2014 by authors and Scientific Research Publishing Inc.

This work is licensed under the Creative Commons Attribution International License (CC BY).

http://creativecommons.org/licenses/by/4.0/

(c) (i) Open Access

\section{Abstract}

In this paper, a numerical study is reported based on the Finite Element Method (FEM) and the Finite Integral Technique (FIT) of Ansoft's HFSS and CST Microwave Studio (MWS) formulations, respectively, applied to a Bended Complementary Split-Ring Resonator (BCSRR) of rejection band extending from 4.1 GHz to 4.6 GHz. The proposed BCSRR structure is combined with the design of a circularly polarized cylindrical antenna array of square patches with trimmed opposite corners. The performance of the cylindrical antenna array is characterized and compared to that of the flat profile. It is found that the proposed BCSRR reduces the mutual coupling to -15 dB between two patches with a separation of only $1 / 11$ th free-space wavelength for applications involving Multi Input Multi Output (MIMO) system.

\section{Keywords}

BCSRR; Cylindrical Array; MIMO

\section{Introduction}

Recent developments in MIMO systems for applications involving biomedical devices, personal and portable equipment [1], raised the demand for conformal RF wireless and electronic devices [2]. Concurrently, metamaterials have witnessed rapid developments in wireless communications, particularly for antennas [3] and passive RF devices [4]. However, conformal MTM structures are still in their early research phase [3].

*Corresponding author. 
In [5], the behavior of an individual Complementary Split-Ring Resonator (CSRR) and the coupling between several CSRRs were reported numerically and experimentally. Furthermore, the performance of tunable triangular split ring resonator and wire strip structures of concentric and non-concentric configurations were reported in [6]. A planar metamaterial structure of spiral elements which provides multiple left-handed modes was proposed in [7] and compared against the classical split-loop spiral arrays. The effects of gap number and position in split ring resonators of a single rectangular geometry with one, two and four gaps were studied in [8]. The authors in [9] investigated the characteristics of band gaps using split ring structures in terms of band gap width with respect to the resonance frequency. Finally, the effects of the orientation of the split ring resonators with respect to both electric and magnetic fields on the frequency resonances were reported in [10].

In this paper, a numerical study conducted to examine the performance of a circularly polarized antenna array in comparison to the flat profile with the objective of studying the effect of involving the CSRR on the radiation pattern. The rest of the paper is organized as follows. In Section 2, the geometrical details of the BCSSR and antenna array structures are presented. The performance of the BCSRR and antenna array is reported in Section 3. Finally, we conclude the paper in Section 4.

\section{BCSRR and Antenna Array Geometry}

\subsection{BCSRR Geometry}

CSRRs provide harmonic rejection bands which can be useful in wireless devices of both flat and conformal profiles. This structure is introduced in our design as EBG defects in the ground plane for the proposed cylindrical array. The traditional SRR couples with the vertically component of the magnetic field. However, the CSRR, based on the duality, interacts with the vertically polarized electric field [9]. The proposed CSRR consists of two concentric split rings. The dimension of the outer ring is $5.7 \mathrm{~mm}$ and the inner is $5.1 \mathrm{~mm}$ with a trace of space $0.3 \mathrm{~mm}$ in width as shown in Figures 1(a) and (b). The proposed CSRR is bended on a cylinder, $14 \mathrm{~mm}$ in diameter, as can be seen in Figure 1(b).

\subsection{Antenna Array Geometry}

The antenna element is a square patch with coroners-truncated at the opposite ends to provide circular polarization as seen in Figures 2(a) and (b). The patch is bended on a polyimide cylindrical substrate. The thickness of the substrate is $0.5 \mathrm{~mm}$ and the relative dielectric constant is 3.5 and a loss tangent of 0.004 as shown in Figures 2(c) and (d). The ground plane is defected by four BCSRR inclusions as depicted on Figure 2(c). The patch elements are separated with $\lambda / 11$, where $\lambda$ is the free-space wavelength at $4.42 \mathrm{GHz}$. The patch elements are excited with $50 \Omega$ discreet port. Mutual coupling reduction is achieved by folding the patch elements on a cylindrical profile and defecting the ground with conformal BCSRR structures to direct the electromagnetic radiation in the opposite side from the neighbor patch element and reject the surface wave propagation, respectively.

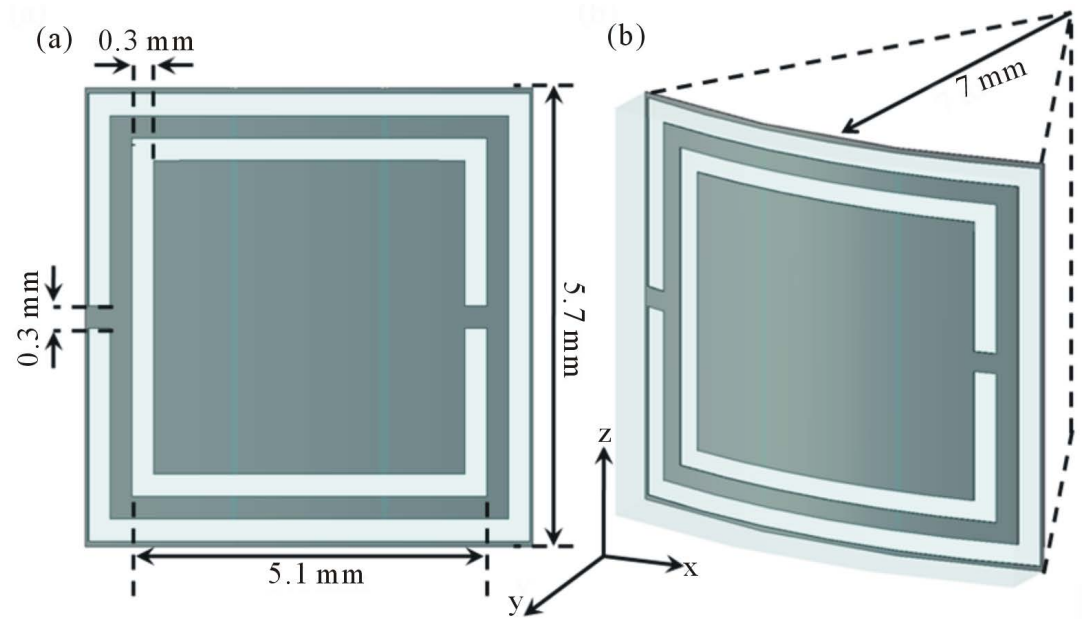

Figure 1. CSRR geometry: (a) Flat profile and (b) Bended profile. 


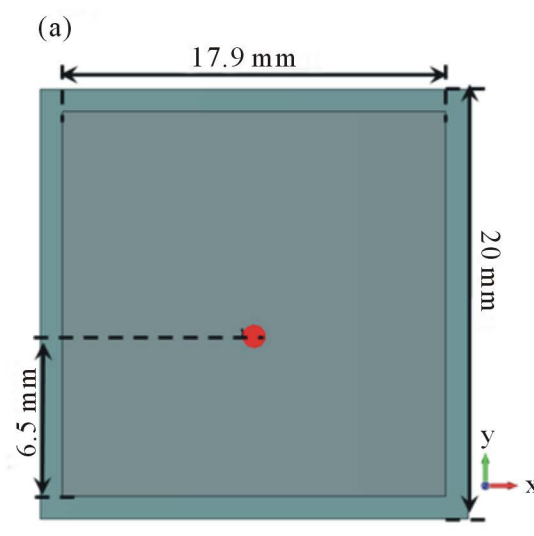

(b)

$3 \mathrm{~mm}$

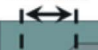

(c)

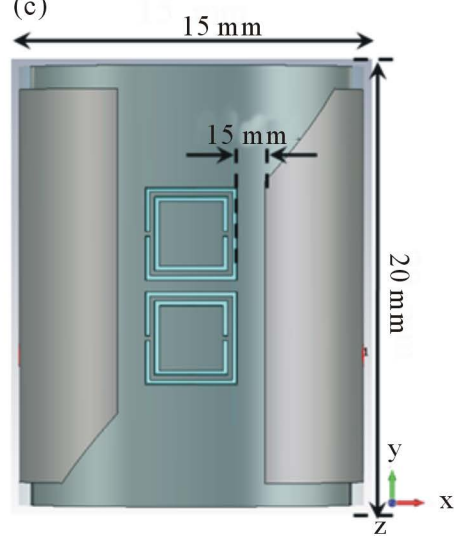

(d)

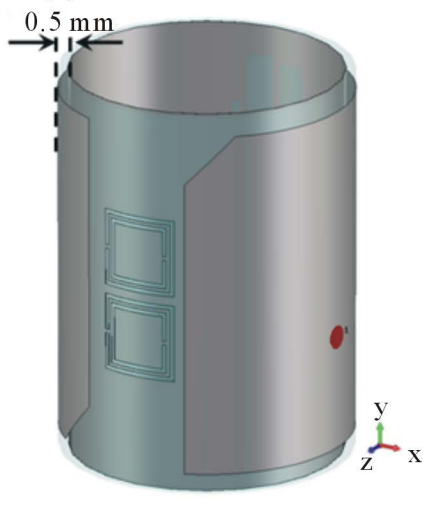

Figure 2. Antenna array geometry: (a) and (b) patch geometry, (c) and (d) front and perspective views of the antenna array.

\section{Numerical Results and Discussion}

In this section, the performance of the BCSRR and the antenna array is discussed numerically using HFSS based FEM and validated using FIT of CST formulations as follows:

\subsection{BCSRR Performance}

In this section, results from eigenmode analysis is performed using Ansoft's HFSS [11] are presented to demonstrate the dispersion diagram of the BCSRR. The corresponding dispersion diagram of the BCSRR, for the first and second mode, is plotted along the $\Gamma-\chi$ axis, from $0^{\circ}$ to $180^{\circ}$. As seen in Figure 3, the first bandgap zone is observed from $4.1 \mathrm{GHz}$ to $4.6 \mathrm{GHz}$.

\subsection{Antenna Array Performance}

A comparison between the folded and the planar profiles in terms of $S_{11}, S_{12}$, and radiation pattern are performed in this section. It is found that the cylindrical profile exhibits a reduction in the frequency resonance of about $150 \mathrm{MHz}$ as seen in Figure 4(a). Moreover, with the cylindrical profile, $\lambda / 11$ as a separation distance between patches is sufficient to reduce the mutual coupling to $-15 \mathrm{~dB}$, while, it is not less than $\lambda / 4$ for the flat profile to get the same reduction as can be seen in Figure 4(b). Furthermore, the mutual coupling reduction in the cylindrical profile is achieved with only 4 BCSRR as compared to six CSRR for the flat profile.

The radiation patterns, in the $E$ - and $H$-planes, are evaluated at $4.42 \mathrm{GHz}$ and $4.57 \mathrm{GHz}$ for both cylindrical and flat profiles, respectively, as seen in Figure 5. The cylindrical profile exhibits an observable enhancement in the bore-sight gain and the beam width in comparison to the flat profile without significant change in the radiation pattern. This enhancement in the bore-sight gain is achieved by reducing the back lobe due to the introduc- 


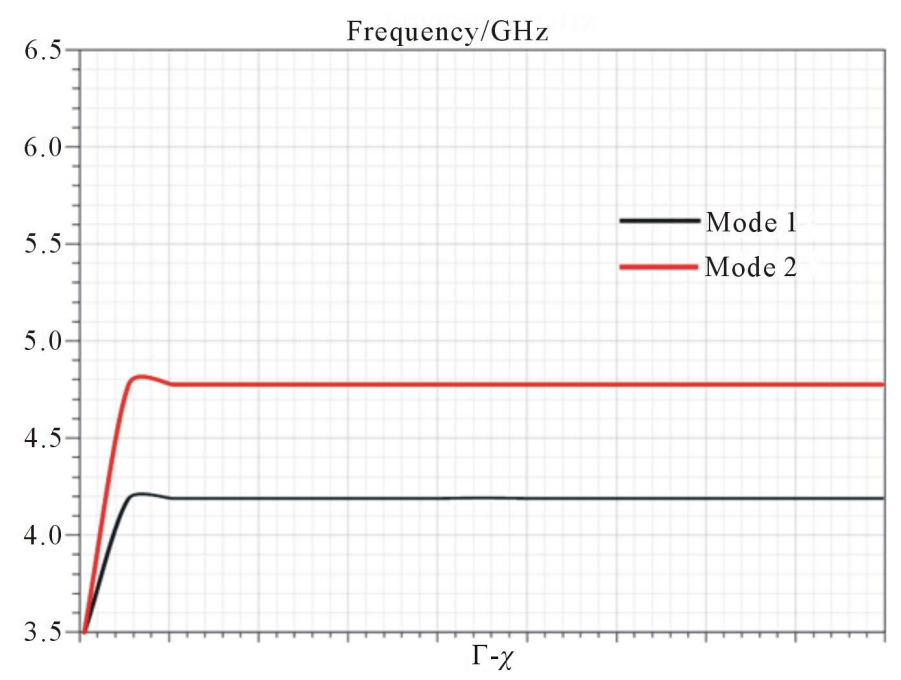

Figure 3. Dispersion diagram for BCSRR unit cell.
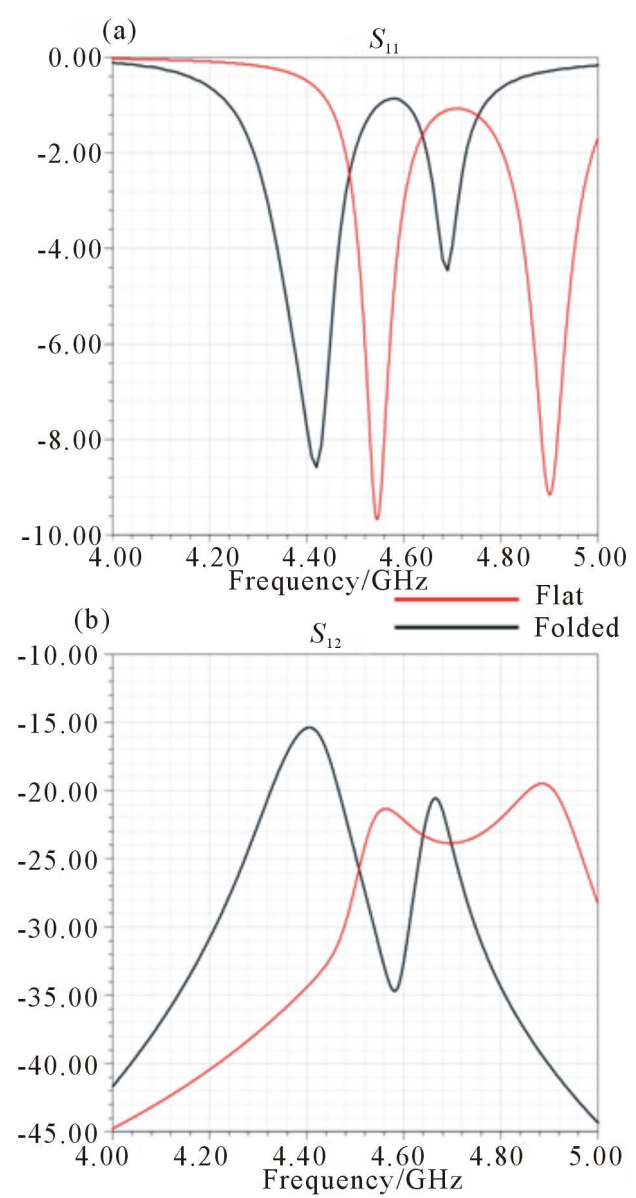

Figure 4. $S$-parameters: (a) $S_{11}$ and (b) $S_{12}$.

tion of the BCSRR. The back lobe is increased in the flat profile after introducing the CSRR. Table 1 summarizes the performance of the antenna array in both cylindrical and the flat profiles.

For a MIMO system with two antenna elements, the envelope correlation between the antenna elements is given by [12] 


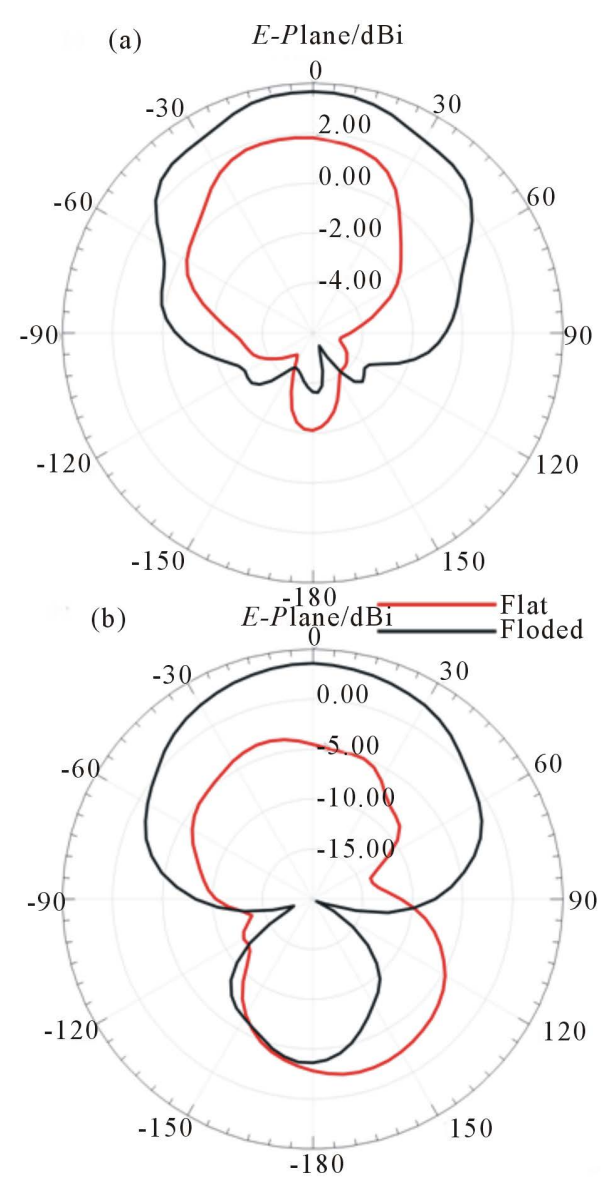

Figure 5. Radiation patterns; (a) E-plane and (b) $H$-plane.

Table 1. The antenna array performance.

\begin{tabular}{ccccccc}
\hline Profile & Frequency resonance/GHz & $S_{11} / \mathrm{dB}$ & $S_{12} / \mathrm{dB}$ & Gain/dBi & Beam width & Axial ratio \\
\hline Folded & 4.42 & -9 & -15 & 5.2 & $140^{\circ}$ & 1.75 \\
Flat & 4.57 & -10 & -22 & 4.5 & $87^{\circ}$ & 2.32 \\
\hline
\end{tabular}

$$
\rho_{e}=\frac{\left|S_{11}^{*} S_{12}+S_{21}^{*} S_{22}\right|^{2}}{\left[1-\left(\left|S_{11}\right|^{2}+\left|S_{21}\right|^{2}\right)\right]\left[1-\left(\left|S_{22}\right|^{2}+\left|S_{12}\right|^{2}\right)\right]}
$$

The correlation envelop is shown in Figure 6 for both cylindrical and flat profiles. The cylindrical antenna array exhibits a correlation factor of 0.08 at $4.42 \mathrm{GHz}$. The correlation factor of the flat antenna array is 0.05 at $4.57 \mathrm{GHz}$.

\subsection{Numerical Validation}

The performance of the cylindrical antenna array is re-evaluated using CST MWS [13] and compared to the simulation results obtained from HFSS to verify the accuracy of obtained results and to validate the design concept. An excellent agreement has been found between the results obtained from MWS and HFSS, which supports the results for the proposed antenna array. Figure 7(a) shows the frequency spectra of $S_{11}$ obtained both software packages. A slight discrepancy is found in the obtained results from CST MWS with a shift in resonant frequency from $4.42 \mathrm{GHz}$ back to $4.2 \mathrm{GHz}$. The reduction in the magnitude of $S_{12}$ is increased as can be ob- 
served in Figure 7(b). The radiation patterns in $E$ - and $H$-plane are almost the same, however, the bore-sight gain increased with $0.25 \mathrm{dBi}$ for the results obtained from CST in comparison to HFSS as can be seen in Figure 8.

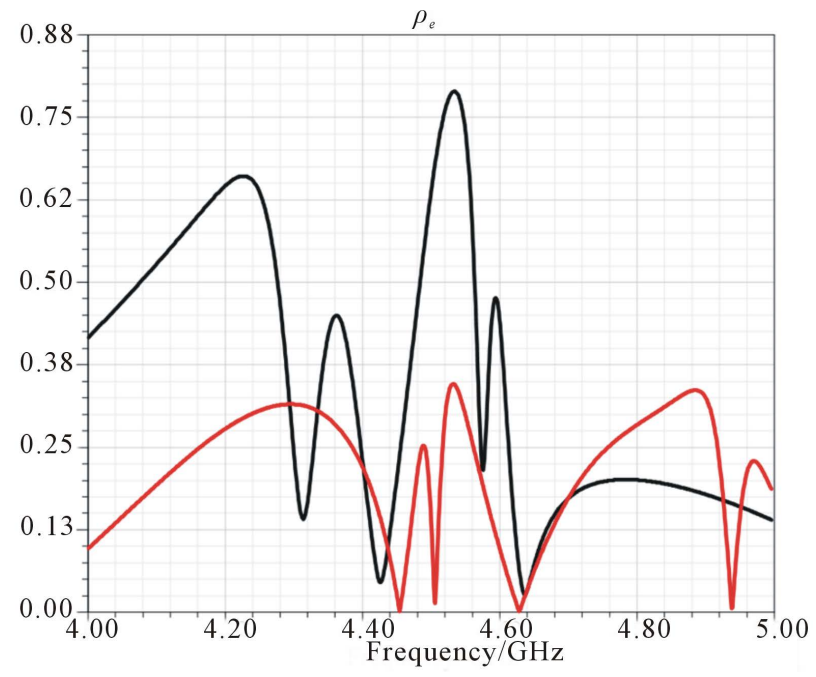

Figure 6. Correlation envelop.
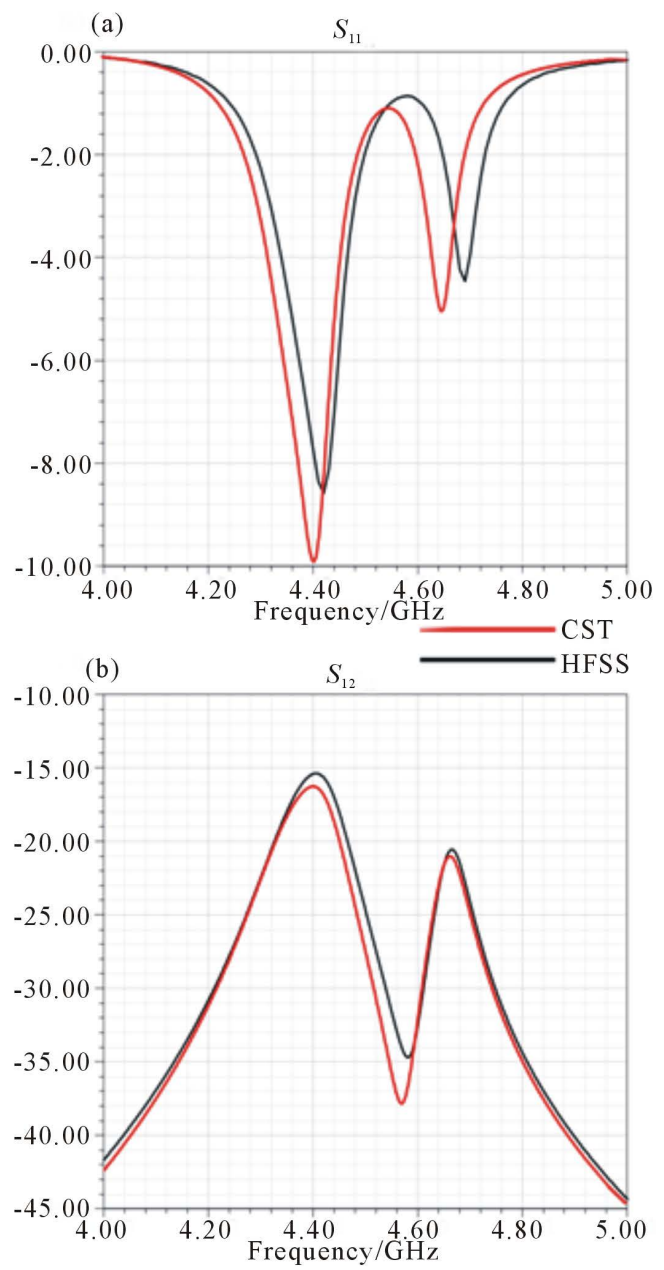

Figure 7. S-parameters; (a) $S_{11}$ and (b) $S_{12}$. 


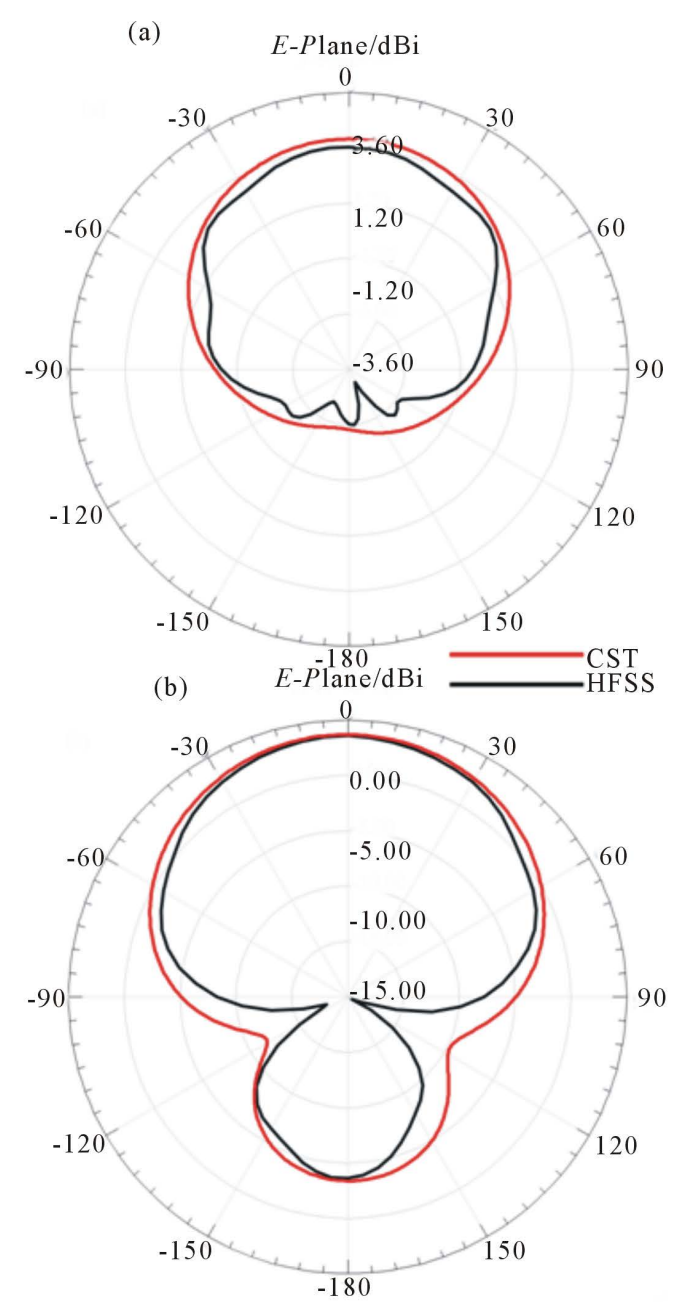

Figure 8. Radiation patterns; (a) E-plane and (b) $H$ plane.

The conducted simulations have been launched with a number of mesh cells $N_{x}=1030, N_{y}=1105$, and $N_{z}=$ 1705 along the $x, y$, and $z$ axes, respectively, and a time step of 3.7067594908 ns. The mesh of HFSS consists of 455,682 tetrahedrals.

\section{Conclusion}

A numerical study based on FEM and FIT of HFSS and CST MWS formulations, has been conducted to CSRR defects when subjected for bending on a folded ground plane of a cylindrical antenna array for MIMO devices. The proposed BCSRR shows a rejection band at $4.3 \mathrm{GHz}$ which is the fundamental resonance mode of the proposed antenna. It is found that the proposed structure exhibits insignificant effects on the radiation patterns of the microstrip antenna in comparison to the sever effects with the flat profile. An observable enhancement in the bore-sight gain and the beam width is achieved with respect to the flat profile. The mutual coupling is reduced to $-15 \mathrm{~dB}$ with a separation distance of $\lambda / 11$. Two different numerical approaches are conducted to validate the obtained results for the proposed antenna array. Finally, an excellent agreement has been achieved between the obtained results from HFSS and CSTMWS that support the validity and the accuracy of our design.

\section{References}

[1] Sun, Y. and Rogers, J.A. (2007) Inorganic Semiconductors for Flexible Electronics. Advanced Materials, 19, 18971916. http://dx.doi.org/10.1002/adma.200602223 
[2] Mi, M., Edwards, L., Bashir, R.M. and Khattak, S. (2008) Wearable Flexible Multi-Band Antenna Based on a Square Slotted Printed Monopole. IEEE Antennas Propagation Conference, 1, 397-400.

[3] Zhu, S. and Langley, R. (2009) Dual-Band Wearable Textile Antenna on an EBG Substrate. IEEE Transactions on Antennas and Propagation, 57, 962-935. http://dx.doi.org/10.1109/TAP.2009.2014527

[4] Cui, T.J., Smith, D. and Liu, R. (2003) Metamaterials: Theory, Design, and Applications. John Wiley \& Sons, Hoboken.

[5] Niu, J.X. and Zhou, X.L. (2007) Analysis of Balanced Composite Right/Left Handed Structure Based on Different Dimensions of Complementary Split Ring Resonators. Progress in Electromagnetics Research, 74, 341-351. http://dx.doi.org/10.2528/PIER07051802

[6] Sabah, C. (2010) Tunable Metamaterial Design Composed of Triangular Split Ring Resonator and Wire Strip for sand c-Microwave Bands. Progress in Electromagnetics Research, 66, 341-357. http://dx.doi.org/10.2528/PIERB10051705

[7] Guo, Y. and Xu, R. (2006) Planar Metamaterials Supporting Multiple Left-Handed Modes. Progress in Electromagnetics Research, 66, 239-251. http://dx.doi.org/10.2528/PIER06113001

[8] Penciu, R.S., Aydin, K., Kafesaki, M., Koschny, T., Ozbay, E., Economou, E.N. and Soukoulis, C.M. (2008) MultiGap Individual and Coupled Split-Ring Resonator Structures. Optical Society of America, 16, 1-14.

[9] Balmaz, P.G. and Martin, O.J.F. (2002) Electromagnetic Resonances in Individual and Coupled Split-Ring Resonators. Journal of Applied Physics, 92, 2929-2936. http://dx.doi.org/10.1063/1.1497452

[10] Marqués, R., Mesa, F., Martel, J. and Medina, F. (2003) Comparative Analysis of Edge- and Broadside-Coupled Split Ring Resonators for Metamaterial Design-Theory and Experiments. IEEE Transactions on Antennas and Propagation, 51, 2572-2581. http://dx.doi.org/10.1109/TAP.2003.817562

[11] (2011) Ansoft’s High Frequency Structure Simulator HFSS. 13th Version. http://www.ansoft.com

[12] Blanch, S., Romeu, J. and Corbella, I. (2003) Exact Representation of Antenna System Diversity Performance from Input Parameter Description. Electronics Letters, 39, 705-707. http://dx.doi.org/10.1049/el:20030495

[13] (2011) CST Microwave Studio. 13th Version. http://www.cst.com 